\title{
Organizational Learning for Intelligence Amplification Adoption: Lessons from a Clinical Decision Support System Adoption Project
}

\author{
Fons Wijnhoven ${ }^{1}$ (D)
}

Accepted: 8 September 2021 / Published online: 9 October 2021

(c) The Author(s) 2021

\begin{abstract}
Intelligence amplification exploits the opportunities of artificial intelligence, which includes data analytic techniques and codified knowledge for increasing the intelligence of human decision makers. Intelligence amplification does not replace human decision makers but may help especially professionals in making complex decisions by well-designed human-AI system learning interactions (i.e., triple loop learning). To understand the adoption challenges of intelligence amplification systems, we analyse the adoption of clinical decision support systems (CDSS) as an organizational learning process by the case of a CDSS implementation for deciding on administering antibiotics to prematurely born babies. We identify user-oriented single and double loop learning processes, triple loop learning, and institutional deutero learning processes as organizational learning processes that must be realized for effective intelligence amplification adoption. We summarize these insights in a system dynamic model—containing knowledge stocks and their transformation processes—by which we analytically structure insights from the diverse studies of CDSS and intelligence amplification adoption and by which intelligence amplification projects are given an analytic theory for their design and management. From our case study, we find multiple challenges of deutero learning that influence the effectiveness of IA implementation learning as transforming tacit knowledge into explicit knowledge and explicit knowledge back to tacit knowledge. In a discussion of implications, we generate further research directions and discuss the generalization of our case findings to different organizations.
\end{abstract}

Keywords Analytics · Clinical decision support system · Intelligence amplification adoption · Organizational learning · System dynamics

\section{Introduction}

Intelligence amplification (IA) aims at making people smarter with the use of artificial intelligence (AI) (Rouse \& Spohrer, 2018; Schmidt, 2017a, 2017b; Xia \& Maes, 2013). These AI technologies include image, speech and natural language processing, and problem solving and recommender systems that are more or less rule-based or machine learning systems (Rouse, 2020). Recent work in AI also has explored the idea of computational intelligence which is “... a set of nature-inspired computational methodologies and approaches to address complex problems associated with real world applications to which traditional methodologies

Fons Wijnhoven

a.b.j.m.wijnhoven@utwente.nl

1 Faculty of Behavioural, Management and Social Sciences, University of Twente, P.O. Box 217, 7500 AE Enschede, The Netherlands and approaches are ineffective or infeasible. It primarily includes fuzzy logic systems, neural networks and evolutionary computation" (Sugumaran et al., 2017, p. 969). Many interesting technical developments in AI have been reported, some of the special issue of Information Systems Frontiers edited by Sugumaran et al. (2017) but the social perspective of organizational contexts and user-AI interactions that make these technological developments have value in practice have been as yet under-researched (Harper, 2019). Therefore, this article takes a social perspective on AI adoption, with a focus on developing knowledge to let human-AI interactions be effective, which we call an organizational learning perspective (Crossan et al., 1999).

In intelligence amplification, AI has an indirect effect on actual human decisions as a consequence of keeping the human in the loop of AI outcome quality checking, interpretation, and maintenance of these systems (Grønsund \& Aanestad, 2020). Therefore, the actual AI effects on decisions are the outcomes of difficult to predict individual 
and organizational learning processes (Schmidt, 2017b) in interactions of human learning and machine learning, which Seidel et al. name triple loop learning (Seidel et al., 2018). A possible direct effect of artificial intelligence, where computers take over human decision making, is often infeasible in professional contexts because of the required decision accountability, the problem ambiguity, and the decisional uncertainty involved (Eberhardt et al., 2012; Etzioni \& Etzioni, 2017). The simpler cases that are highly repetitive and involve not much diversity of sources and insights, could be run by rule-based or expert system, but many professional cases, like medical and legal use contexts, require an understanding of a personal situation and thus the application of contextually embedded knowledge "... grounded in the situated sociohistoric contexts of our lives and work" (Orlikowski, 2006, p. 460).

Artificial intelligence technologies may become supportive agents in an intelligence amplification socio-technical system involving one or more professional decision makers. Such a socio-technical system may support the process of understanding a case by the re-use and sharing of codified knowledge or the mining of new knowledge by data analytics (Markus, 2001). These socio-technical systems use and produce emerging knowledge because of ongoing learning processes (Nonaka, 1994; Orlikowski, 2006). This makes a static variance or causal analysis of the impact of intelligence amplification less relevant and requires the logic in such studies to be replaced by an emergent impact paradigm (Markus \& Robey, 1988; Markus et al., 2002). This changes the assessment of intelligence amplification from researching the contribution of IA adoption (e.g., reduced mortality) as a dependent variable to the embedding of the technology with needs and the context of the stakeholders in a sociotechnical system. Such embedding does not only require the understanding of its use for specific decision situations, i.e., a user-oriented learning process, but also the development of institutional environments as scaffolds of learning that both constrain and enable IA to be developed and implemented. These scaffolds “... include physical objects, linguistic systems, technological artifacts, spatial contexts, and institutional rules-all of which structure human activity by supporting and guiding it, while at the same time configuring and disciplining it" (Orlikowski, 2006, p. 462).

In more detail, we describe IA adoption challenges here for a clinical IA system for the purpose of learning about IA adoption as an organizational learning process. Such a clinical IA system contains multiple AI techniques, like visualisation (Turkay et al., 2014), zooming, sorting, and filtering of relevant patient data, images and video recordings (Korotkov \& Garcia, 2012) and analysis of data from multiple sources like the patient's medical record and genomic data for diagnosis and treatment selection (Raghupathi \& Raghupathi, 2014). Natural language processing (NLP) can also be used as part of a clinical decision support system (CDSS) to extract the meaning from written notes in medical records (Auffray et al., 2016; Holzinger et al., 2008; Ivanović $\&$ Budimac, 2014). A CDSS thus is a collection of artificial intelligence systems for decision making in a clinical context. A CDSS like Watson for Oncology incorporates a form of prescriptive analytics by ranking treatment alternatives along predicted effectiveness for a given diagnosis with the support of mining knowledge from over 600 medical journals, hundreds of different medical data sources, and statistical evidence (Somashekhar et al., 2018). Such a CDSS may also be part of an intelligence amplification systems as a secondary knowledge mining system, i.e.,“....analysts attempt to extract knowledge from records that were collected by others, possibly unknown to the reuser, for very different purposes" (Markus, 2001, p. 71). Markus (2001) states that secondary knowledge mining requires extensive datamining skills, knowledge and experience, but many more capabilities must be developed to make this effective for intelligence amplification.

The literature on medical CDSS has reported on many challenges in realizing intelligence amplification. McNut et al. (2016) and Rumsfeld et al. (2016) mention that for the fields of oncology and cardiovascular diagnoses and treatments respectively, CDSS need sufficiently large and reliable datasets, which are not always available. For realizing such large data sets hospitals will have to share their data (Dinov, 2016; Peek et al., 2014; Salas-Vega et al., 2015). However, a lack of systems interoperability and different data taxonomies prevent inter-hospital data sharing (Asokan \& Asokan, 2015; Raghupathi \& Raghupathi, 2014). Medical practitioners are also concerned regarding the accuracy of classifications and predictions when the data sets are too small (Budhiraja et al., 2016; Geerts et al., 2016; Kruse et al., 2016; Szlezak et al., 2014) and the algorithms used are often non-transparent or incomprehensible (Eberhardt et al., 2012; Maia et al., 2017). Intransparency of algorithms may result in unacceptable feelings of loss of reasoning control for medical professionals (Eberhardt et al., 2012; Holden \& Karsh, 2010; Maillet et al., 2015). Experiences with unreliable registrations in patient files also contribute to mistrust in CDSS (Salas-Vega et al., 2015). Many of the reasons for not trusting and resisting CDSS are thus not technical or psychological (i.e., the medical professionals risk perception) but are rooted in ethical, legal, and managerial intelligence amplification requirements being insufficiently met (Abouelmehdi et al., 2017; AndreuPerez et al., 2015). Coping with these IA challenges thus requires specific capabilities, including personal skills and emergent organizational and inter-organizational capabilities and thus we explore IA adoption as an organizational learning process.

This article aims at theoretical generalization [i.e., theory formalization of grounded insights (Glaser \& Strauss, 2009)] 
from a CDSS IA development case and by this create an organizational learning theoretical foundation for IA development in organizations. The kind of theory that we realize here is an analytic theory, which "... provides a description of the phenomena of interest, analysis of relationships among those constructs, the degree of generalizability in constructs and relationships and the boundaries within which relationships, and observations hold" (Gregor, 2006, p. 619). By this theory, researchers and practitioners will be able to disentangle the complexity of human-AI interactions in processes of IA implementation and will be able to design systems. Organizational learning has been described in its foundational literature as the creation of improvements (i.e., single loop learning) or innovations (i.e., double loop learning) and the creation of norms, rules and conditions by which these knowledge creation processes may be done best (so-called deutero learning or institutional learning) (Crossan, 2003; Visser, 2007; Wijnhoven, 2001). Such learning processes are emergent knowledge creation processes that transform intuitive or tacit insights into more explicit knowledge that can be combined to larger collections of explicit knowledge that next have to be integrated with everyday tacit understanding (Nonaka, 1994). Following the insight that we can know more than we can tell (Polanyi, 1966), the single loop, double loop and deutero learning processes start and end with tacit knowledge of individuals that may be shared in groups, networks or organizations. Clearly, only parts of tacit knowledge can be externalized, i.e., made person-independent and re-usable. In shared work practitioners contexts (Markus, 2001), much of the knowledge that cannot effectively be explicated, like specific person-dependent skills and experiences, can be registered and shared as part of the group's transactive memory (Majchrzak et al., 2013). Such transactive memory thus does not codify and store the knowledge content itself but contains a shared memory for group members of who has what knowledge and information, enabling a shared division of cognitive labor (Brandon $\&$ Hollingshead, 2004). Alternatively, the explicit knowledge created by secondary knowledge miners may be materialized in databases, decisions rules and data mining pattern outcomes with different levels of technological readiness (Mankins, 2009; Rouse, 2020; Russell \& Norvig, 2016).The explicit knowledge that is created in the process of realizing the organizational conditions for IA may be materialized in (privacy) laws, inter-organizational data sharing standards, and IT management policies. Externalization thus first places implicit knowledge on a human independent medium like an information system. Second, this explicit knowledge can be combined with other explicit knowledge, e.g., by the creation of data warehouses, analytics outcomes, and rulebased expert systems. Third, the more advanced combined knowledge can produce recommendations to decision makers that decision makers can internalize by integrating it with personal experiences, values, and skills, i.e., tacit knowledge. Finally, professionals may discuss problems and recommendations with colleagues for verification and to learn from each other or develop new standards and guidelines via a learning process called socialization. Socialization learning has group tacit knowledge and transactive memory as combined tacit knowledge outcomes. Similar knowledge emergent processes also exist for the deutero learning process that set the rules, plans and conditions for single or double loop learning in the IA context.

This brings us to the following research question:

What challenges exist in the socialization, externalization, combination, and internalization stages of intelligence amplification adoption?

The next section further describes the organizational learning approach to IA, after which we explain the case methodology used for detecting relevant adoption challenges. Section four presents our results of a medical IA adoption case and section five discusses the generalization of these results for other professional non-medical contexts. Lastly, the sixth section entails the conclusion and reflects about further research.

\section{Intelligence Amplification Adoption as Organizational Learning}

The information systems field has extensively discussed IT adoption. One approach here is the "theory of innovation adoption" which is grounded in Rogers (2010) work and describes innovation adoption as a multi-stage process of awareness development, persuasion, decision to adopt, implementation and continuation of use. These stages include learning processes where the person collects information first of where the innovation is about, next to decide about adopting, and finally collecting information for deciding on continuation of adoption and possible more extensive use. The innovation process affects five categories of adopters that represent different levels of technology adoption in the social system. These groups are named innovators, early adopters, early majority, late majority, and laggards, and they differ on their willingness and ability to take varying levels of risks with innovation adoption decisions. Innovators are willing to take risks, have the highest social status, have financial liquidity, and thus can absorb decision failures, and have closest contact to scientific sources and other innovators. Their risk tolerance allows them to adopt technologies that may ultimately fail. Laggards are the last to adopt an innovation. Individuals in this category show little to no opinion leadership. These individuals typically have an aversion to change-agents. Laggards typically tend to be focused on "traditions", lowest social status, lowest financial liquidity, oldest among adopters, and in contact with only 
family and close friends. In a social system, the distribution of members in each category may follow a bell shape with innovators and laggards being the smallest groups and the early majority and late majority being the largest groups. Clearly, innovation adoption is not a simplistic calculation of costs and benefits or the collection of information for making such a calculation. It also involves social pressure, norms and values, and personal risk and prestige. In AI adoption, some professionals may want to be innovators or early adopters for generating prestige where others will be laggards and risk averse. For being an innovator or early adopter, one needs to have the resources and reputation to be able to act as such (Burke et al., 2009).

Technology acceptance models have been developed to further identify personal or environmental factors that influence an individual's adoption decisions. Venkatesh et al. (2003) have identified eight models of technology acceptance: the theory of reasoned action, technology acceptance model (TAM), motivation theory model, theory of planned behavior (TPB), a combined model of TAM and TPB, a model of PC utilization, innovation diffusion theory as grounded in Rogers's work, and social cognitive theories of technology adoption. They combined these theories in a unified theory of acceptance and use of technology (UTAUT) (Venkatesh et al., 2003). The UTAUT holds that the key constructs that explain use intention and actual information technology use are (1) performance expectancy, (2) effort expectancy, (3) social influence, and (4) use facilitating conditions. Performance expectancy and effort expectancy are both used by people for making an innovation adoption cost benefit calculation. Innovators have higher performance expectations of innovations than laggards. Together with possible social influences like group pressures, norms and values, and active advertising, people will form a level of usage intention, which in its turn may influence actual usage behaviour. Facilitating conditions may be given to individuals for using a technology, like financial support, support expertise, a high-quality usage infrastructure, and thus also touches policies and infrastructures that are created and offered by the institutional contexts of potential adopters (Hull \& Lio, 2006). This means that in contrast to expectations and social influence that influence an individual's intention to use, poor facilitation may turn a person with a high intent to usage to an actual non-user. Gender, age, experience, and voluntariness of use are posited to moderate the impact of the four explanatory key constructs on use intention and behaviour.

Venkatesh et al. (2003) found in a longitudinal study that UTAUT accounts for $70 \%$ of the variance in behavioural intention to use IT and about 50\% in actual use. More recent work of Parasuraman and Colby (2015) offers a technology readiness index which measures optimism and innovativeness as motivating predispositions for IT adoption, and discomfort and insecurity as inhibitors of IT adoption. Both these variance studies, however, do not explain how use intentions and actual use can be developed as part of an awareness creation, persuasion and motivation process for the creation of use intentions and actual use (Markus \& Robey, 1988; Wheeler, 2002). AI adoption has many organizational consequences and therefore the development of knowledge for user adoption is a critical organizational learning process that requires socialization, externalization, combination, and integration of relevant knowledge for adoption decisions. For making the broader IA adoption decisions, the decision makers have to create knowledge of different kinds in multiple learning steps. First the decision maker has to become aware of possibilities and thus to externalize insights in explicit notions of performance and efforts for making a cost-benefit calculation (Rogers, 2010). Because decision making for IA adoption mostly requires the collaboration of work practitioners (Markus, 2001), the decision makers have to socialize and agree on values before such a costs benefit calculation can be made. The outcome of cost-benefit calculations has to be related with existing opportunities and constraints that are to some extent materialized in existing infrastructures, other collateral systems, IT policies and rules, resulting in a combination of explicit knowledge of several kinds. For the actual implementation of the decision, the system has to be internalized by changing existing ways of thinking and working of individual users. Especially for IA systems, the need for understanding AI outcomes and having AI well embedded in processes and people's way of working is vital to let it work well as a decision support tool (Orlikowski, 2006). Finally, all people involved in use and support of the IA system will have to evaluate it effectiveness for realizing shared knowledge and beliefs to further use or extend its use by repeating the learning cycle starting at a more extended level of awareness. These learning cycles in the context of artificial intelligence use are not only human learning processes, but also involve machine learning, single loop and double loop, and the integration of machine learning outcomes with human learning outcomes, named triple loop learning (Seidel et al., 2018).

Because IA adoption is the adoption of AI technologies for professional decision making, IA adoption happens in and for an organization context. The learning processes therefore are organizational, and organizations may enable or constraint the IA adoption learning process in several ways. Organizations also may learn to influence the single loop, double loop and triple loop learning processes. These learning enabling and constraining processes are named deuteron learning (Visser, 2007; Wijnhoven, 2001). Deutero learning involves the development of dynamic capabilities, i.e., the continuous building up of resources for innovation (Kraaijenbrink et al., 2010). The essence of the dynamic capabilities (DCP) development is a continuous organizational effort 
of acquiring and reconfiguring capabilities for staying in tune with changing markets and technologies (Lavie, 2006). Similarly, intelligence amplification requires a capability of learning to find out how AI can be deployed effectively, i.e., the creation of norms, processes and scaffolds for individual organization members to learning to adopt AI and develop an IA socio-technical system. Such norms and processes are both enablers and constraints. Wheeler (2002) describes how IT related capabilities can be created via a Net-Enabled Business Innovation Cycle (NEBIC). This theory describes the cycle of value creation in net-enabled organizations through four dynamic capabilities: (1) choosing emerging/ enabling technologies, (2) matching with economic opportunities, (3) executing business innovation for growth, and (4) assessing customer value. The relations between these capabilities are processes that describe learning from each of the four capabilities, communicating the results to the following capability, and feeding back market-based metrics. Translated to a more general theory of IA adoption, the choosing capability includes routines to create insights on emerging and enabling technologies that could support IA-enabled value creation. These insights could relate to, for example, mobile technologies, Internet technologies, and AI technologies and their technological readiness levels (TRL) (Mankins, 2009). The choosing capability has a broader focus than just technology. Choosing a technology goes together with selecting a technical platform and ecosystem and will be done at an IT policy level as well as the professionals view on its potential, like with organizational learning it involves the explication of feelings of usefulness for the organization. The resulting choosing insights are input to the matching capability, which includes routines to combine these insights with concrete business process activities and strategic goals of organizations. The matching capability, i.e., a combination learning activity, aims at revealing new IT-enabled economic opportunities. When these opportunities are detected, the next step is to internalize the detected opportunity insights by integrating them with existing processes and mindsets and finally to evaluate its success in terms of customer and organizational values, i.e., to create new group tacit knowledge. Each step requires a stage gate to be passed as an outcome of an organizational learning process performed by stakeholders with outputs consisting of individual's intention to use and facilitating conditions that are created for actual usage.

The Nonaka model of organizational learning states that through socialization people share tacit knowledge and create a group level tacit knowledge. This group tacit knowledge contains facts and beliefs that people share but also norms, values, and standards. The externalization process transforms tacit knowledge into explicit and codified knowledge. This kind of knowledge is placed on a person independent "material" medium, like books, databases, manuals, and recipes. Via the combination process, content of these person-independent media can be combined to a larger set of combined knowledge. Such combined knowledge can consist of data warehouses and larger knowledge systems like digital encyclopaedias and recommender systems. The internalization process translates (group) explicit knowledge back into a personal understanding by integrating the explicit knowledge components, like recommendations, calculations, and reasoning, with what the individual person thinks is appropriate in the specific decision context. Thus, in internalization the explicit knowledge is evaluated on ethical, logical, and common-sense beliefs. Knowledge thus develops by a continuous emergent process of socialization, externalization, combination, and internalization. For IA adoption, this learning process thus is both user-oriented single, double, and triple loop learning and an institutional deutero learning process involving the development of enablers, like motivators, capabilities and resources, and constraints for these individual learning processes. Figure 1 gives a variant of Nonaka's SECI model to summarize these learning processes as a system dynamic process, which means that knowledge stocks (the boxes in the model) receive growth from a learning process that delivers an inflow of new knowledge (wide arrows in Fig. 1). These inflows are more voluminous or speedily if they reuse insights from previous stocks represented by the thin broken link arrows that inserts the stock knowledge in the learning process and the deutero enabling or constraining variables (represented by the ovals in Fig. 1). (For more information about system dynamics modelling, the reader is referred to (FortmannRoe, 2014)). Note that we assume no unlearning as we have no outflows from the knowledge stocks. This means that we see the learning as continuously increasing the knowledge needed for adoption Also note that externalization involves the codification of existing knowledge, whereas combination may result in new insights, thus double loop learning. The triple loop learning, i.e., the learning from the AI systems and integrating these new insights in the stock of knowledge, only happens after the AI-based recommendations are delivered to the decision maker in the internalization process. In the socialization process, participants in this process may share existing (single loop learning) and new knowledge (double loop learning learning). The deutero learning only involves the influencing of these learning processes. Note that some authors have equated deutero learning with triple loop learning in the past, but we follow Seidel et al. (2018) who reserve the term triple loop learning for the integration of human and machine learning processes, and we reserve the term deutero learning for learning to manage the organizational learning processes (Visser, 2007; Wijnhoven, 2001). 
Fig. 1 The IA adoption learning process

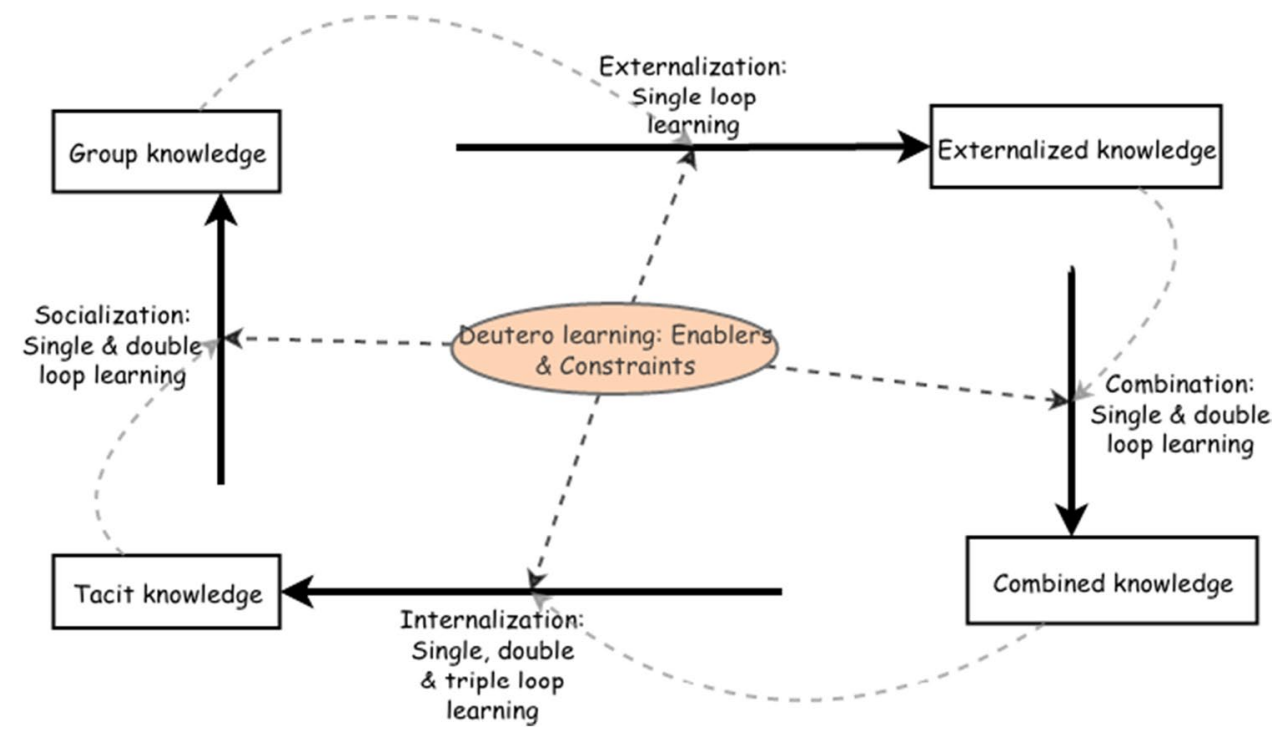

\section{Methodology}

\subsection{Case Study and Context}

This research wants to describe the organizational learning challenges for a concrete implementation of an IA system and to generate a generalizable formal theory out of these grounded insights (Glaser \& Strauss, 2009). To realize this, we use a rich case description in which key stakeholders were interviewed regarding their view on the adoption of an AI-based CDSS (Klein Koerkamp, 2019). This case is from a University Medical Centre, which initiated the "Analytics for Medicine" program in spring of 2017 to enable personalized healthcare with data analytics. This is a hospital-wide program with a special team of clinicians and data scientists. The case focuses on a CDSS pilot named Sepsis Identification Speed, which was run by the Neonatology department. Babies that are born too early are sensitive to infection. Treatments with invasive procedures, such as intravenous lines, blood samples and ventilation are all potential entry points for bacteria and add to the risk of illness. The Neonatology department wants to know as early as possible if a patient will become ill and what treatment is most appropriate. The current care process is as follows: (1) the physician suspects an infection, (2) the physician takes a blood culture, (3) this blood culture is examined for bacteria, and (4) when the culture is positive, the blood is examined by gram staining and bacteria are coloured to make them visible under the microscope to identify the species. This process can take up to 48 hours which can be crucial in the development of the infection and the administration of antibiotics.

The CDSS aims to support the physicians when they consider administering antibiotics. The CDSS focuses on predicting with a minimum of false negatives. False negatives are the most dangerous situations because a false-negative advises not to give antibiotics when it is needed. But to complicate the decisions, following false positives is harmful as well, because administering antibiotics has some negative side effects as well. The single loop learning process focusses on speeding up the predictions and decision process, where the double loop learning process aims at reducing prediction error. The CDSS uses different data sources from the database of neonatology which consists of 6000 children born between 24 and 32 weeks. This data originates from several systems whose data must be integrated and prepared within data management before analysis. For increasing its prediction reliability, the hospital develops cooperation with other hospitals to generate a larger dataset for analytics. The model development method applied for analysis is the 'gradient boosting' technique of predictive machine learning.

\subsection{Data Collection}

The units of analysis in this case study are the different CDSS project stakeholders' opinions. Besides of the directly intended medical users, we identify political and financial stakeholders, IT support stakeholders, vendor developers, clinical ethicists, clinical methodologists, and regulator stakeholders. Based on this analysis, the respondents shown in Table 1 were selected as the units of observation in the case study. The respondents were directly approached, visited at an event, and selected and approached from the vendor's network and advise from other interviewees. Some interviewees have overlapping stakeholder roles as can be seen in Table 1.

The case study used semi-structured interviews which provide openings for a narrative to unfold while also 
Table 1 Interviewee numbers, descriptions, and stakeholder roles

\begin{tabular}{lll}
\hline $\mathrm{Nr}$ & Respondent description & Stakeholder role \\
\hline $\mathrm{R} 1$ & Current CEO of a hospital cluster in the UK & Political \& financial \\
R2 & Research program director e-health \& big data & Political \& financial \\
R3 & Business developer of e-health platform & Political \& financial \\
R4 & Physician and clinical owner the CDSS & Medical user \\
R5 & Education director health informatics & Political \& financial \\
R6 & Physician and clinical director & Medical user \\
R7 & Healthcare director of the vendor & Political \& financial \\
R8 & Senior technical consultant of the vendor & Developer \\
R9 & System engineer healthcare of the vendor & Developer \\
R10 & Ex-physician, senior vendor healthcare systems & Political, financial, \& medical user \\
R11 & Ex-physician and data scientist of the hospital & Medical user \& developer \\
R12 & Physician and clinical owner of CDSS & Medical user \\
R13 & Program manager data analytics & Political \& financial \\
R14 & CEO consultancy firm healthcare & Political \& financial \\
R15 & Managing partner CDSS developer & Developer, political \& financial \\
R16 & Hospital ethicist, member ethical commission & Ethicist \\
R17 & Business engineer CDSS developer & Developer \& maintenance operator \\
R18 & Hospital methodologist & Methodologist \\
R19 & Inspector e-health of the government (IGJ) & Regulator \\
R20 & Ex-physician and analytics entrepreneur & Medical user \& developer \\
R21 & Hospital IT/ICT manager & Operational support \\
R22 & Clinical CEO notified body & Regulator \\
R23 & National manager of hospital data registrations & Regulator \\
\hline
\end{tabular}

applying questions based on the literature review (Galetta, 2013). The unscripted narrative enabled the researcher to explore the respondents' view. Interviewees were asked to describe features, components, processes, or activities of the CDSS in which barriers for implementation may occur. Moreover, after mentioning the barriers, interviewees were asked about possible ways to meet them in the shorter or longer run.

\subsection{Data Analysis}

The data from the semi-structured interviews have been recorded and transcribed. Subsequently, the transcribed statements have been coded with the ATLAS.ti qualitative data analysis software. To ensure the research reliability, the transcribed interview has been sent to the specific interviewee with the request to supply feedback and corrections where needed (Whittemore et al., 2001). The total meaning coverage of the used concepts [i.e., content validity (Babbie, 2015)] within the interviews is assessed by cross checking the concepts in the questionnaires with the healthcare $\&$ technology experts of the CDSS software vendor. The author of this article next classified relevant interview statements by organizational learning categories socialization, externalization, combination, and integration and in more depth selected indications of IA adoption challenges as deuteron learning challenges. The external validity includes the inductive generalization of the CDSS case by a comparison with different IA contexts to be analyzed in the section after the case results presentation. Generalization here is thus an inductive formalization of grounded insights (Glaser \& Strauss, 2009).

\section{Results: CDSS Adoption Challenges}

This section presents data for the four organizational learning categories of the SECI sequence, which emphasizes the importance of tacit knowledge as the start and the end of any organizational learning. All externalizations and combinations that may follow from the learning process are reflections or extensions of tacit understandings but they are also inputs for new tacit understandings. For each SECI learning process, we describe the stakeholder view by presenting relevant statements and possible deuteron influencing variables.

\subsection{Socialization Challenges}

Socialization is the sharing of tacit knowledge among people. Tacit knowledge may be any insight or experience that is not formally defined and highly person-dependent. R7 
(healthcare director vendor): "Hospitals have (...) a cultural problem, the physicians are too distant from the IT department." Involving physicians within the development stage of the CDSS will be beneficial for trust and acceptancy of it. Therefore, R11 (ex-physician and data scientist) says: "It is important to bring along a group of physicians within this process or else you will get the 'not invented by me syndrome'."

Including a regulatory expert in the $R \& D$ team might be beneficial to the $\mathrm{CE}^{1}$ approval process preparation of the CDSS project team. R22 (Clinical CEO notified body) says: "A regulatory professional within a $R \& D$ team involved from step one can think of what Notified Body or food and drug administration (FDA) market approval conditions are". According to R19 (inspector e-health), the EU is currently developing norms for artificial intelligence which are also applicable to CDSS. These norms could also give a greater legal framework. R22 (global clinical director notified body) says: "The inspection of healthcare and representatives of other EU member states validated the new (...) procedures during joined assessments. Very often during these joint assessments the EU member state representatives had different interpretations of the regulations. Hence, it took 7 years to write the new legislation, then you have a meeting with representatives of a notified body from several countries and the healthcare inspection and they are still discussing what the rules exactly say."

Thus, the socialization process is not just of physicians and their teams, but also with technical, ethical, quality control (inspection) and legal stakeholders. All must understand each other. In this process mutual influencing is of course happening.

\subsection{Externalization Challenges}

Externalization is the transformation of tacit knowledge to explicit knowledge by codification of rules and knowledge and the registration of events and facts for managing them on person-independent media. The term "knowledge" is used here in a broad sense in the many ways that people say to know something, and thus includes facts and figures, theories and explanations, methods and skills, and experiences (Mingers, 2008).

Some interviewees say that the CDSS adoption is challenging because of several externalizations needed. For example, R5 (professor health informatics) says: "I believe that a lot of professionals are not satisfied with the registration load....". R1 (ex-chairman board of the hospital board) says: "There is a tension between how we can

\footnotetext{
${ }^{1} \mathrm{CE}$ stands for Conformité Européenne and is the European Union quality certification label.
}

standardize the systems input and how can we keep the input requirements user friendly". Even when these data would be available and correct, a lot of data processing and analytics is needed which is not always compliant with CE quality norms. R11 (ex-physician and data scientist) says: "Some data producing machines within the hospital are validated and CE approved for research and not for healthcare, which is needed for CDSS, a new CE approval of the data warehouse is required to realize this transition."

Physicians also hold professional expectations that make them hesitant to work with a CDSS. R20 (ex-physician and analytical entrepreneur) says: "CDSS technology enforces strict working according to guidelines and thus may deprive physicians from their sense of added value. This (...) makes physicians feel less valuated." This is not necessarily a defensive attitude but could be based on sincere professional doubts. R17 (Business engineer and CDSS developer) says: "It is not clear if the improvement of care is worth the costs of data scientists, medical trial, infrastructure and maintenance of the CDSS project."

A CDSS should use randomized control trial (RCT) outcomes, but R4 (clinical owner CDSS) says: “... randomized experiment where half will be exposed to the algorithm and the other half not (...) is seriously hard (...), we have to look at how we can show the clinical relevance without a randomized study."

Thus, the externalizations needed for a realizing a working AI system are seen as risky in several ways and cannot easily convince medical professionals and hospital managers to choose in favour of them.

\subsection{Combination Challenges}

Combination is the process of bringing different externalized parts (like databases and applications) together in one larger system. R12 (clinical owner CDSS) says: "All the data the CDSS requires can be extracted from the hospital wide critical data layer for analytical processing by means of an API call. (...) This data layer is currently under construction, technologically feasible, however, realization depends on commitment and budget."

Such a data layer would automatically collect, integrate and prepare the data from different data sources, but there is a lack of data sharing protocols. R8 (Senior technical consultant vendor) says: "Every system generates its own data in a way that is most easy for this system. Which is not wrong of course. Only when you want to join the data from these systems, you might realize that you should have done it in a different manner."

.... and R12 (clinical owner CDSS) says: "There is no automated process that collects data for analysis and makes it available to other solutions, all the data is still in 
Fig. 2 Intelligence amplification organizational learning challenges

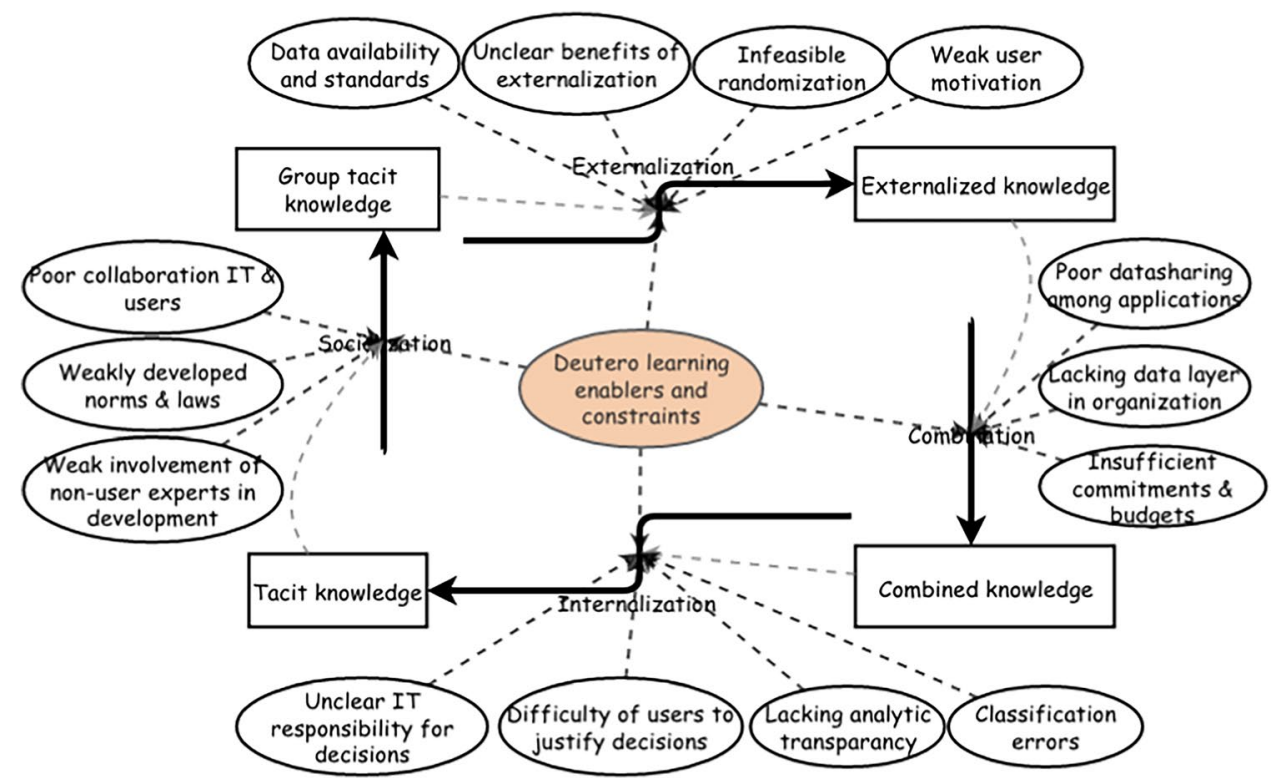

its original source and has to be extracted and integrated manually by somebody to enable the following analysis."

Thus, the CDSS has to be viewed in the context of other data sources and systems and for this a well working policy, systems architecture, and standards have to be developed.

\subsection{Internalization Challenge}

Internalization is the process of humanly interpreting knowledge, insights and recommendations given by a system. For making recommendations acceptable for decision makers, they have to rely on transparent data analysis procedures. R10 (ex-physician and sales manager vendor) says: "The analytical process often requires transparency. (...) If a physician stands in front of a judge, he/she must be able to exactly explain how the process was executed." ..... And the system must be compliant with the standards for CE approval. R12 (clinical owner CDSS) says: "However, the system still misclassifies some patients because they had other symptoms or something special, only 6 of the 500 children. This is not much, however, if all 6 babies die, this is hard to explain, of course." And R13 (Program manager data analytics) says: "It does not matter if the algorithm says to intervene or not to intervene, the physician is always responsible and therefore is hesitant in using these systems." Whereas the responsibility for medical decisions lies with the physician, there is a moral responsibility that lies with the developer. R16 (Ethicist and member of the medical ethical commission) says: "If an algorithm makes a mistake (...) the developer is partially responsible since s/he built this into the algorithm.".
Thus, medical decisions in this case have high life impact and thus medical professionals only want to use the CDSS of its performance expectation is arguably very high.

\section{Case Analysis}

Figure 2 summarizes the CDSS development challenges mentioned in the case as deuteron learning constraints and enabler variables that influence the CDSS socialization, externalization, combination, and internalization processes. We further analyze the challenges for each of these processes while trying to generalize the insights of the case to general IA challenges per learning process. In a grounded theory development effort this is named the formalization of theory (Glaser \& Strauss, 2009). We will do this by asking the question of what is specific for a health context case and thus also answer the question on what issues the CDSS case is not representative for many other IA adoption contexts. To develop a theory of IA adoption, we thus identify besides of the 4 explanatory variables of intention to use and actual use (Venkatesh et al., 2003) a sequence of organizational learning processes in terms of deutero learning variables, i.e., learning enabling and constraining conditions and contextual variables on which IA adoption projects may differ for their learning processes.

The socialization process in which stakeholders of different disciplines develop a consensus on how to proceed in the IA project is critical for later IA adoption learning. In medical contexts, the knowledge input from lawyers regarding European and national laws on principles for privacy, data property, patient rights, safety, and quality of decisions explaining the need for their development, is a 
sufficient reason for medical professionals not (yet) to want to be engaged in the project, despite a vendor's motivation to explain the opportunities. This also points at an important contextual factor for medical IA systems: law and regulation. Because medical professionals act on people's life and health, a good legal context for this is probably more important than for other professions like education, software development, or architecture (Willmott et al., 2016).

In the process of further externalizing how the CDSS could look like, medical professionals may still have doubts regarding the quality and cost-benefit of such a system for medical professionals. This means that after the legal issues are settled, the system should be at the proper technological readiness level (Mankins, 2009). This also may require substantial testing before being considered for use and further development or implementation. Software testing is without doubt important for any information system, but for medical devices the technical readiness level must be very high because any risk taking in use may be a risk with again large human and legal consequences (Yusif et al., 2017).

If this externalization hurdle could be taken, the proposed system could be integrated with other systems and the broader organizational and inter-organizational landscape. For this, hospitals need sometimes extra budget and resources, which only can be acquired if the IA system meets a positive organizational cost-benefit calculation. Such cost-benefit calculation has to show items like time saved by professionals, increased hospitals incomes, or less costs of treatments. All these variables are often more difficult to assess for medical contexts (Henshall et al., 2012; Husereau et al., 2014). But a better treatment also has to cover its cost from an organizational perspective. So this makes IT investment decisions in the medical field sometimes very difficult (Castlen et al., 2019; Lakdawalla et al., 2017).

When the combination hurdle has been taken, the IA system can be implemented and given for use and the internalization hurdle comes next. For internalization, the medical professional must become convinced that recommendations from an AI system can be truly trusted and that the medical decision is thus better backed with state-of-the-art knowledge then without. Here, the medical field deviates again from other fields. Although any professional must be trustworthy, for a medical professional it is less easy to say that an erroneous decision was taken because a system recommended it (Eberhardt et al., 2012; Grote \& Berens, 2020). This is especially the case when decisions are very complex, and an optimum choice option is hard to find. In these cases also the patient involvement in the decision making may be larger because patients have to bear the consequences of choices made (Charles et al., 1999). In such situations, an AI system may be less valuable, and in actual use the preferences, norms and values of stakeholders will determine much of the actual choice. Clearly, the medical field differs from most other professional disciplines by the large trust and the high personal responsibilities that need backing. Legal expert systems are especially well known for their rule-based character, as their knowledge is not probabilistic and cannot so easily be based on large numbers of nearequivalent cases (Leith, 2016). In the field of education, educational analytics are an upcoming field but still very new (Macfadyen, 2017). In engineering, rule-based systems may be more common as the natural scientific laws with which engineers work have a highly reliable and stable rule-based nature (Bullon et al., 2017; Savage et al., 2019) But also in these non-medical contexts, AI systems trust is an important value in IA for professionals.

If the internalization hurdle is taken, the learning process can continue to further steps, like the implementation of more features or the role out of the system to more professional users. At each moment that a hurdle cannot be taken, the process may go a step back, and the knowledge content and transactive memory that was build up can be usefully taken as a basis for further development.

\section{Conclusions and Implications}

This study realized an understanding of many challenges of artificial intelligence adoptions in the broader context of intelligence amplification development as an organizational learning process. More specifically, we described four organizational learning processes for IA adoption, starting with a tacit knowledge stock and finishing with an enhanced tacit knowledge stock by which this approach articulates IA as a socio-technical environment for making people become smarter. These learning outcomes can be incremental single loop improvements or more radical double loop learning innovations. The learning stocks do not only inform individual adoption decisions but also exist to realize the group learning and the organization-wide learning that are essential for letting AI systems deliver value. These learning processes are emergent and continuous, facilitated, speeded up or delayed by deutero learning variables. In our CDSS case, high requirements for legal backing, patient risk life avoidance, and technical-scientific maturity of the CDSS and its outcomes showed to be essential deuteron learning variables. Non-medical contexts may need less strict development on rules and laws, may be satisfied with systems of a lower technological readiness, even prototypes, may require less (inter-)organization wide arrangements because they can be used on an individual basis, and the professional decisions made with AI could be less affecting the rights and lives of individuals. These empirical propositions are in need of research in non-medical professional case contexts.

These insights have practical implications for the design of IA implementation projects. The project developer should 
assess the context, different learning stages and the thresholds that should be overcome per learning process. Given the complexities involved and the active involvement, contribution and commitments of multiple stakeholders, such projects should be management by more senior project managers who use the deutero learning variables for speeding up the process, moving the process to wanted directions, and allow profiting from new learning during the project's execution (Pich et al., 2002). The need for learning during project executions has been articulated in modern agile project management methods before (Campanelli \& Parreiras, 2015), but IA implementation projects demand also learning outside of the technical focus of a project. IA implementation project managers should be able to develop a development team as well as acquire financial and other resources from organization internal and external stakeholders. Project managers should be aware that the learning in IA implementations is not only human or technical single or double loop, but also triple loop, i.e., developing the interactions of AI learning and human learning with mutual influences (Seidel et al., 2018). This triple loop learning requires a careful gradual development of capabilities not only of computer scientists involved but of (professional) users as well (Grønsund \& Aanestad, 2020). The end to such a learning process may be difficult to define as long as triple loop learning generates new insights. Besides of triple loop learning, the practice of IA implementations may also require the development of new organizational norms (i.e., deutero learning) that enable triple loop learning, e.g., the development of IT stakeholders' responsibilities in decisions and the development of well working collaborative IT and user teams.

Theoretically and academically, this approach gives a revitalization of the importance of tacit knowledge stocks as the alpha and omega of organizational learning (Nonaka, 1994) and shows the relevance of this insight in the context of IA systems. This is in line with previous understanding of the difference between artificial or computational intelligence (Sugumaran et al., 2017) and intelligence amplification as a way of increasing human thinking power (single loop or double loop) with artificial intelligence as part of it (Berrar et al., 2013). However, more knowledge is needed of the diversity of organizational contexts and how these interact on the IA adoption process design and realization. For this, we suggest a number of research directions along different organizational learning processes.

Regarding deutero learning, one may for example ask the question if learning norms in other organizations than (academic) medical will be much different and what the influence of learning norms is on different challenges of IA implementation. Learning norms are part of the broader concept of organizational culture (Hofstede, 1980). Previous survey research on the impact of hospital organizational culture on IT adoption in general (do Carmo Caccia-Bava et al., 2006) has identified that different corporate cultural elements like an organization's internal versus external orientation and the level of flexibility or control influences and organization's technological absorptive capacity and via that the success of IT adoptions in hospitals. We propose an update of this study by more depth towards the type of IT to be adopted, especially AI technologies, and the learning processes of users and other organizational stakeholders and with a comparison with other non-medical organizations, if survey data can be acquired. Also UTAUT survey studies (Venkatesh et al., 2003) can be extended and made more specific for IA implementation motivations and norms, including organizational learning as process and dependent variables. Such survey studies can be helpful in further understanding differences of IA implementations in contexts and give insights for project managers on how to identify, prioritize and handle the relevant deutero variables.

Regarding triple loop learning, the interactions of human users and AI systems can be studied as single loop or double loop learning processes. The difference between single loop and double learning is important and comparable to control and innovation (Argyris \& Schon, 1978) or exploitation and exploration (March, 1991). Much previous research has been dedicated to the effectiveness of intelligent amplification for control (for example Althuizen et al., 2012; Bensoussan et al., 2009) but new research may be needed on using IA for innovation. Research may be useful to find out if IA can really make people smarter by generating new insights, new views, and creative solutions. Such research will have to go more in depth on creativity and how human creativity can be extended or improved with IA systems (Ardaiz-Villanueva et al., 2011; Rouse \& Spohrer, 2018). Because creativity is often generated through interactions of multiple team members, one may also open research on triple loop learning as not only an individual human and system interaction, but also as interactions between multiple persons using multiple systems for innovation. In our theoretical framework this implies a focus on combination and socialization learning as part of IA implementation. Whereas combination has been studied before in the context of enterprise systems development (Strong \& Volkoff, 2010), our case study demonstrates that combination is also a complex organizational process of developing standards and IT policies. Research on how socialization is both a start and end to IA implementation should answer research questions like how people develop motivations, norms and values for IA adoption and how new AI system usage influences people in their decisions and collaboration, and how people will maintain being the alpha and omega of knowledge in organizations.

Acknowledgements This article is the outcome of multiple reflections on the AI adoption case described by my previous student Rick Klein Koerkamp, who I supervised during his case study. A previous version 
of the article has been reviewed and presented at the ICTO 2019 conference on the impact of artificial intelligence via which multiple suggestions for improvement have been gained before being submitted to this journal. I want to especially thank ICTO chair Antoine Harfouche for supporting me to realize this article. I also thank anonymous reviewers and the editor of this journal for multiple comments resulting in substantial revisions again, making the article as it is now. The full responsibility for the article's content, however, remains with the author.

Open Access This article is licensed under a Creative Commons Attribution 4.0 International License, which permits use, sharing, adaptation, distribution and reproduction in any medium or format, as long as you give appropriate credit to the original author(s) and the source, provide a link to the Creative Commons licence, and indicate if changes were made. The images or other third party material in this article are included in the article's Creative Commons licence, unless indicated otherwise in a credit line to the material. If material is not included in the article's Creative Commons licence and your intended use is not permitted by statutory regulation or exceeds the permitted use, you will need to obtain permission directly from the copyright holder. To view a copy of this licence, visit http://creativecommons.org/licenses/by/4.0/.

\section{References}

Abouelmehdi, K., Beni-Hssane, A., Khaloufi, H., \& Saadi, M. (2017). Big data security and privacy in healthcare: A review. Procedia Computer Science, 113, 73-80. https://doi.org/10.1016/j.procs. 2017.08.292

Althuizen, N., Reichel, A., \& Wierenga, B. (2012). Help that is not recognized: Harmful neglect of decision support systems. Decision Support Systems, 54(1), 713-728. https://doi.org/10.1016/j. dss.2012.08.016

Andreu-Perez, J., Poon, C. C. Y., Merrifield, R. D., Wong, S. T. C., \& Yang, G.-Z. (2015). Big data for health. IEEE Journal of Biomedical and Health Informatics, 19(4), 1193-1208. https://doi.org/10. 1109/JBHI.2015.2450362

Ardaiz-Villanueva, O., Nicuesa-Chacón, X., Brene-Artazcoz, O., de Acedo, S., Lizarraga, M. L., de Acedo, S., \& Baquedano, M. T. (2011). Evaluation of computer tools for idea generation and team formation in project-based learning. Computers \& Education, 56(3), 700-711. https://doi.org/10.1016/j.compedu.2010.10.012

Argyris, C., \& Schon, D. (1978). Organizational learning: A theory of action approach. Addison Wesley.

Asokan, G. V., \& Asokan, V. (2015). Leveraging "big data" to enhance the effectiveness of "one health" in an era of health informatics. Journal of Epidemiology and Global Health, 5(4), 311-314.

Auffray, C., Balling, R., Barroso, I., Bencze, L., Benson, M., Bergeron, J., Bernal-Delgado, E., Blomberg, N., Bock, C., Conesa, A., Del Signore, S., \& Zanetti, G. (2016). Making sense of big data in health research: Towards an EU action plan. Genome Medicine, 8(71), 2-13. https://doi.org/10.1186/s13073-016-0323-y

Babbie, E. R. (2015). The basics of social research (7th ed.). Cengage Learning.

Bensoussan, A., Mookerjee, R., Mookerjee, V., \& Yue, W. T. (2009). Maintaining diagnostic knowledge-based systems: A control-theoretic approach. Management Science, 55(2), 294-310. https://doi. org/10.1287/mnsc. 1080.0908

Berrar, D., Konagaya, A., \& Schuster, A. (2013). Turing test considered mostly harmless. New Generation Computing, 31(4), 241-263. https://doi.org/10.1007/s00354-013-0401-2

Brandon, D. P., \& Hollingshead, A. B. (2004). Transactive memory systems in organizations: Matching tasks, expertise, and people. Organization Science, 15(6), 633-644.
Budhiraja, R., Thomas, R., Kim, M., \& Redline, S. (2016). The role of big data in the management of sleep-disordered breathing. Sleep Medicine Clinics, 11(2), 241-255.

Bullon, J., González Arrieta, A., Hernández Encinas, A., \& Queiruga Dios, A. (2017). Manufacturing processes in the textile industry. Expert systems for fabrics production. ADCAIJ: Advances in Distributed Computing and Artificial Intelligence Journal, 6(4), 15-23. https://doi.org/10.14201/ADCAIJ2017641523

Burke, M. A., Fournier, G. M., \& Prasad, K. (2009). The diffusion of a medical innovation: Is success in the stars? further evidence. Southern Economic Journal, 75(4), 1274-1278.

Campanelli, A. S., \& Parreiras, F. S. (2015). Agile methods tailoringA systematic literature review. Journal of Systems and Software, $110,85-100$.

Castlen, J. P., Cote, D. J., \& Broekman, M. L. D. (2019). The ethics of funding innovation: Who should pay? Ethics of innovation in neurosurgery (pp. 75-82). Springer.

Charles, C., Gafni, A., \& Whelan, T. (1999). Decision-making in the physician-patient encounter: Revisiting the shared treatment decision-making model. Social Science \& Medicine, 49(5), 651-661.

Crossan, M. (2003). Chris Argyris and Donald Schön's organizational learning: There is no silver bullet. Academy of Management Perspectives, 17(2), 38-39.

Crossan, M. M., Lane, H. W., \& White, R. E. (1999). An organizational learning framework: From intuition to institution. Academy of Management Review, 24(3), 522-537. https://doi.org/10. 5465/amr.1999.2202135

Dinov, I. D. (2016). Methodological challenges and analytic opportunities for modeling and interpreting Big Healthcare Data. GigaScience, 5(12), 2-15. https://doi.org/10.1186/ s13742-016-0117-6

do CarmoCaccia-Bava, M., Guimaraes, T., \& Harrington, S. J. (2006). Hospital organization culture, capacity to innovate and success in technology adoption. Journal of Health Organization and Management, 20(3), 194-217. https://doi.org/10.1108/1477726061 0662735

Eberhardt, J., Bilchik, A., \& Stojadinovic, A. (2012). Clinical decision support systems: Potential with pitfalls. Journal of Surgical Oncology, 105(5), 502-510.

Etzioni, A., \& Etzioni, O. (2017). Incorporating ethics into artificial intelligence. The Journal of Ethics, 21(4), 403-418. https://doi. org/10.1007/s10892-017-9252-2

Fortmann-Roe, S. (2014). Insight maker: A general-purpose tool for web-based modeling \& simulation. Simulation Modelling Practice and Theory, 47, 28-45. https://doi.org/10.1016/j.simpat.2014.03. 013

Geerts, H., Dacks, P. A., Devanarayan, V., Haas, M., Khachaturian, Z. S., Gordon, M. F., Maudsley, S., Romero, K., Stephenson, D., \& Initiative, B. H. M. (2016). Big data to smart data in Alzheimer's disease: The brain health modeling initiative to foster actionable knowledge. Alzheimer's \& Dementia, 12(9), 1014-1021.

Glaser, B. G., \& Strauss, A. L. (2009). The discovery of grounded theory: Strategies for qualitative research. Adeline.

Gregor, S. (2006). The nature of theory in information systems. MIS Quarterly, 30(3), 611-642.

Grønsund, T., \& Aanestad, M. (2020). Augmenting the algorithm: Emerging human-in-the-loop work configurations. The Journal of Strategic Information Systems, 29(2), 101614. https://doi.org/ 10.1016/j.jsis.2020.101614

Grote, T., \& Berens, P. (2020). On the ethics of algorithmic decisionmaking in healthcare. Journal of Medical Ethics, 46(3), 205-211. https://doi.org/10.1136/medethics-2019-105586

Harper, R. H. R. (2019). The role of HCI in the age of AI. International Journal of Human-Computer Interaction, 35(15), 1331-1344. 
Henshall, C., Schuller, T., \& Mardhani-Bayne, L. (2012). Using health technology assessment to support optimal use of technologies in current practice: The challenge of "disinvestment." International Journal of Technology Assessment in Health Care, 28(03), 203-210.

Hofstede, G. (1980). Culture and organizations. International Studies of Management \& Organization, 10(4), 15-41.

Holden, R. J., \& Karsh, B. T. (2010). The technology acceptance model: Its past and its future in health care. Journal of Biomedical Informatics, 43(1), 159-172. https://doi.org/10.1016/j.jbi. 2009.07.002

Holzinger, A., Geierhofer, R., Mödritscher, F., \& Tatzl, R. (2008). Semantic information in medical information systems: Utilization of text mining techniques to analyze medical diagnoses. Journal of Universal Computer Science, 14(22), 3781-3795.

Hull, C. E., \& Lio, B. H. (2006). Innovation in non-profit and forprofit organizations: Visionary, strategic, and financial considerations. Journal of Change Management, 6(1), 53-65.

Husereau, D., Marshall, D. A., Levy, A. R., Peacock, S., \& Hoch, J. S. (2014). Health technology assessment and personalized medicine: Are economic evaluation guidelines sufficient to support decision making? International Journal of Technology Assessment in Health Care, 30(02), 179-187.

Ivanović, M., \& Budimac, Z. (2014). An overview of ontologies and data resources in medical domains. Expert Systems with Applications, 41(11), 5158-5166. https://doi.org/10.1016/j. eswa.2014.02.045

Klein Koerkamp, R. M. (2019). The road from analytical CDSS invention to implementation in healthcare. University of Twente. Retrieved from https://essay.utwente.n1/77341/

Korotkov, K., \& Garcia, R. (2012). Computerized analysis of pigmented skin lesions: A review. Artificial Intelligence in Medicine, 56(2), 69-90. https://doi.org/10.1016/j.artmed.2012.08. 002

Kraaijenbrink, J., Spender, J.-C., \& Groen, A. J. (2010). The resourcebased view: A review and assessment of its critiques. Journal of Management, 36(1), 349-372.

Kruse, C. S., Goswamy, R., Raval, Y. J., \& Marawi, S. (2016). Challenges and opportunities of big data in health care: A systematic review. JMIR Medical Informatics, 4(4), e38.

Lakdawalla, D., Malani, A., \& Reif, J. (2017). The insurance value of medical innovation. Journal of Public Economics, 145, 94-102.

Lavie, D. (2006). Capability reconfiguration: An analysis of incumbent responses to technological change. Academy of Management Review, 31(1), 153-174. https://doi.org/10.5465/amr.2006.19379 629

Leith, P. (2016). The rise and fall of the legal expert system. International Review of Law, Computers \& Technology, 30(3), 94-106.

Macfadyen, L. (2017). Overcoming barriers to educational analytics: How systems thinking and pragmatism can help. Educational Technology, 57(1), 31-39.

Maia, A.-T., Sammut, S.-J., Jacinta-Fernandes, A., \& Chin, S.-F. (2017). Big data in cancer genomics. Current Opinion in Systems Biology, 4, 78-84.

Maillet, É., Mathieu, L., \& Sicotte, C. (2015). Modeling factors explaining the acceptance, actual use and satisfaction of nurses using an electronic patient record in acute care settings: An extension of the UTAUT. International Journal of Medical Informatics, 84(1), 36-47. https://doi.org/10.1016/j.ijmedinf.2014.09.004

Majchrzak, A., Wagner, C., \& Yates, D. (2013). The impact of shaping on knowledge reuse for organizational improvement with wikis. MIS Quarterly, 37(2), 455-469. https://doi.org/10.25300/MISQ/ 2013/37.2.07

Mankins, J. C. (2009). Technology readiness assessments: A retrospective. Acta Astronautica, 65(9-10), 1216-1223. https://doi.org/10. 1016/j.actaastro.2009.03.058
March, J. G. (1991). Exploration and exploitation in organizational learning. Organization Science, 2(1), 71-87.

Markus, M. L. (2001). Toward a theory of knowledge reuse: Types of knowledge reuse situations and factors in reuse success. Journal of Management Information Systems, 18(1), 57-93. https://doi. org/10.1080/07421222.2001.11045671

Markus, M. L., Majchrzak, A., \& Gasser, L. (2002). A design theory for systems that support emergent knowledge processes. MIS Quarterly, 26(3), 179-212.

Markus, M. L., \& Robey, D. (1988). Information technology and organizational change: Causal structure in theory and research. Management Science, 34(5), 583-598.

McNutt, T. R., Moore, K. L., \& Quon, H. (2016). Needs and challenges for big data in radiation oncology. International Journal of Radiation Oncology Biology Physics, 95(3), 909-915.

Mingers, J. (2008). Management knowledge and knowledge management: Realism and forms of truth. Knowledge Management Research \& Practice, 6(1), 62-76. https://doi.org/10.1057/palgr ave.kmrp. 8500161

Nonaka, I. (1994). A dynamic theory knowledge of organizational creation. Organization Science, 5(1), 14-37. https://doi.org/10. $1287 /$ orsc.5.1.14

Orlikowski, W. J. (2006). Material knowing: The scaffolding of human knowledgeability. European Journal of Information Systems, 15(5), 460-466. https://doi.org/10.1057/palgrave.ejis.3000639

Parasuraman, A., \& Colby, C. L. (2015). An updated and streamlined technology readiness index. Journal of Service Research, 18(1), 59-74. https://doi.org/10.1177/1094670514539730

Peek, N., Holmes, J. H., \& Sun, J. (2014). Technical challenges for big data in biomedicine and health: Data sources, infrastructure, and analytics. Yearbook of Medical Informatics, 23(01), 42-47.

Pich, M. T., Loch, C. H., \& Meyer, A. D. (2002). On uncertainty, ambiguity, and complexity in project management. Management Science, 48(8), 1008-1023.

Polanyi, M. (1966). The tacit dimension. University of Chicago Press.

Raghupathi, W., \& Raghupathi, V. (2014). Big data analytics in healthcare: Promise and potential. Health Information Science and Systems, 2(1), 3. https://doi.org/10.1186/2047-2501-2-3

Rogers, E. M. (2010). Diffusion of innovations. Simon and Schuster.

Rouse, W. B. (2020). AI as systems engineering augmented intelligence for systems engineers. Insight, 23(1), 52-54.

Rouse, W. B., \& Spohrer, J. C. (2018). Automating versus augmenting intelligence. Journal of Enterprise Transformation, 8(1-2), 1-21.

Rumsfeld, J. S., Joynt, K. E., \& Maddox, T. M. (2016). Big data analytics to improve cardiovascular care: Promise and challenges. Nature Reviews Cardiology, 13(6), 350-359. https://doi.org/10. 1038/nrcardio.2016.42

Russell, S. J., \& Norvig, P. (2016). Artificial intelligence: A modern approach (3rd ed.). Pearson Education Limited.

Salas-Vega, S., Haimann, A., \& Mossialos, E. (2015). Big data and health care: Challenges and opportunities for coordinated policy development in the EU. Health Systems \& Reform, 1(4), 285-300.

Savage, J., Rosenblueth, D. A., Matamoros, M., Negrete, M., Contreras, L., Cruz, J., Martell, R., Estrada, H., \& Okada, H. (2019). Semantic reasoning in service robots using expert systems. Robotics and Autonomous Systems, 114, 77-92.

Schmidt, A. (2017a). Augmenting human intellect and amplifying perception and cognition. IEEE Pervasive Computing, 16(1), 6-10.

Schmidt, A. (2017b). Technologies to amplify the mind. Computer, 50(10), 102-106.

Seidel, S., Berente, N., Lindberg, A., Lyytinen, K., \& Nickerson, J. V. (2018). Autonomous tools and design: A triple-loop approach to human-machine learning. Communications of the ACM, 62(1), $50-57$.

Somashekhar, S. P., Sepúlveda, M. J., Puglielli, S., Norden, A. D., Shortliffe, E. H., Rohit Kumar, C., Rauthan, A., Kumar, N. A., 
Patil, P., Rhee, K., \& Ramya, Y. (2018). Watson for Oncology and breast cancer treatment recommendations: Agreement with an expert multidisciplinary tumor board. Annals of Oncology, 29(2), 418-423. https://doi.org/10.1093/annonc/mdx781

Strong, D. M., \& Volkoff, O. (2010). Understanding organizationenterprise system fit: A path to theorizing the information technology artifact. MIS Quarterly, 34(4), 731-756. https://doi.org/ $10.2307 / 25750703$

Sugumaran, V., Geetha, T. V., Manjula, D., \& Gopal, H. (2017). Guest editorial: Computational intelligence and applications. Information Systems Frontiers, 19(5), 969-974.

Szlezak, N., Evers, M., Wang, J., \& Pérez, L. (2014). The role of big data and advanced analytics in drug discovery, development, and commercialization. Clinical Pharmacology \& Therapeutics, 95(5), 492-495.

Turkay, C., Jeanquartier, F., Holzinger, A., \& Hauser, H. (2014). On computationally-enhanced visual analysis of heterogeneous data and its application in biomedical informatics. Lecture notes in computer science (including subseries lecture notes in artificial intelligence and lecture notes in bioinformatics) (Vol. 8401, pp. 117-140). Berlin: Springer.

Venkatesh, V., Morris, M. G., Davis, G. B., \& Davis, F. D. (2003). User acceptance of information technology: Toward a unified view. MIS Quarterly, 27(3), 425-478. https://doi.org/10.2307/30036540

Visser, M. (2007). Deutero-learning in organizations: A review and a reformulation. Academy of Management Review, 32(2), 659-667. https://doi.org/10.5465/amr.2007.24351883

Wheeler, B. C. (2002). NEBIC: A dynamic capabilities theory for assessing net-enablement. Information Systems Research, 13(2), 125-146. https://doi.org/10.1287/isre.13.2.125.89

Wijnhoven, F. (2001). Acquiring organizational learning norms: A contingency approach for understanding Deutero learning. Management Learning, 32(2), 181-200.

Willmott, L., White, B., Parker, M., Cartwright, C., \& Williams, G. (2016). Is there a role for law in medical practice when withholding and withdrawing life-sustaining medical treatment? Empirical findings on attitudes of doctors. Journal of Law and Medicine, 24, 342-355.

Xia, C., \& Maes, P. (2013). The design of artifacts for augmenting intellect. In: Proceedings of the 4th Augmented Human International Conference (pp. 154-161). ACM.

Yusif, S., Hafeez-Baig, A., \& Soar, J. (2017). e-Health readiness assessment factors and measuring tools: A systematic review. International Journal of Medical Informatics, 107, 56-64.

Publisher's Note Springer Nature remains neutral with regard to jurisdictional claims in published maps and institutional affiliations.

Fons Wijnhoven is an associate professor of knowledge management and information systems at the University of Twente Enschede, Netherlands since 2001. Dr Wijnhoven researches information and cloud services, the adoption of artificial intelligence in organizations, and project management principles for smart industry development. In this context, he has a specific focus on prediction validity for management decision making. For this research he participates in the University of Twente's Digital Society Institute and the Low-Energy Datacenter Center. He teaches on information services, smart industries, and IT project management. Fons has monographs on information management (Routledge, 2009) and information services design (Taylor \& Francis 2012). His work has been published in journals like Decision Support Systems, Internet Research, J of Management Information SystemsJ of the Operations Research SocietyJ of Strategic Information SystemsJ of the American Society on Information Science \& Technology, ACM Journal of Data and Information Quality, and Applied Energy. 